\title{
The Dynamics of NEO Binary Asteroids
}

\author{
D.J. Scheeres \\ Dept. of Aerospace Engineering, The University of Michigan, Ann Arbor, \\ MI 48109-2140, USA, email: scheeres@umich.edu
}

\begin{abstract}
The dynamics of binary Near-Earth objects (NEO) are discussed and a simple model for the study of their dynamics is introduced. Main results on the motion and stability of binary asteroids are reviewed. The effect of perturbations external to the binary system, including solar gravity, solar radiation pressure, and planetary gravity, are considered.
\end{abstract}

Keywords. asteroid, rotation; dynamics, rotation; binary asteroids

\section{Introduction}

Binary NEO exhibit a rich set of dynamics and are exposed to many external and internal perturbations. These include coupling of orbital and rotational angular momentum and energy, effect of non-spheroidal mass distributions on dynamical evolution, solar gravitational perturbations for bodies close to the sun, planetary tides during close approaches, and Solar irradiation effects.

The general dynamical problem of binary asteroids, or binary bodies in orbit about each other and subject to external perturbations, has received considerable study over the years. The relevant studies include investigations of point mass dynamics about non-spherical bodies Chauvineau et al. (1993), Scheeres (1994), Scheeres et al. (1996), Scheeres et al. 1998, point mass dynamics about point bodies incorporating solar gravitational and radiation perturbations Hamilton \& Burns (1991), Scheeres \& Marzari (2002), effect of solar radiation on finite bodies Ćuk \& Burns (2005), motion of two massive bodies about each other Kinoshita (1972), Maciejewski (1995), Scheeres (2002a), Scheeres (2002b), Breiter et al. (2005), and motion of particles about binary asteroids Scheeres \& Bellerose (2005). Recently, a detailed model and associated dynamics of the NEO binary asteroid (66391) $1999 \mathrm{KW}_{4}$ was studied in detail in Ostro et al. (2006), Scheeres et al. (2006). Despite these many studies the general problem still has many challenges that must be addressed, ranging from better constraints and understanding of the dynamical evolution of these systems to basic questions on what the most important physics for the evolution and energy dissipation of these systems are. The goal of the current paper is simply to define the basic dynamical problem of binary asteroids, introduce an ideal model for binary asteroids and present basic results on its dynamics, and identify the most relevant known perturbations acting on these systems and their characteristics.

\section{The General Model}

We first state the most general form of the binary asteroid dynamics problem. These have been derived in an alternate form in Maciejewski (1995) and provide the equations of motion for the relative translational motion between the two components of the binary asteroid and each body's rotational dynamics. We give the current statement as they are 
in a particularly compact form, as given in Scheeres et al. (2006):

$$
\begin{aligned}
\frac{M_{1} M_{2}}{M_{1}+M_{2}} \ddot{r}_{i} & =U_{r_{i}}+F_{i}\left(r, T^{1}, T^{2}, t\right) \\
\dot{H}_{i}^{I} & =-T_{i j}^{I} \epsilon_{j k l} T_{m k}^{I} U_{T_{m l}^{I}}+M_{i}^{I}\left(r, T^{I}, t\right) \\
\Omega_{i}^{I} & =\left(I_{j i}^{I}\right)^{-1} T_{k j}^{I} H_{k}^{I} \\
\dot{T}_{i j}^{I} & =T_{i k}^{I} \Omega_{l}^{I} \epsilon_{k l j} \\
U\left(r, T^{1}, T^{2}\right) & =\mathcal{G} \int_{B^{1}} \int_{B^{2}} \frac{d m^{1} d m^{2}}{\left|r+T^{1} \rho^{1}-T^{2} \rho^{2}\right|}
\end{aligned}
$$

where $i=1,2,3$ denote coordinates and $I=1,2$ denotes the two bodies. Here $r$ is the relative position vector between the two centers of mass expressed in an inertial frame, $M_{I}$ denotes the mass of body $I, H^{I}$ is the inertial frame angular momentum vector of the $I$ th body, $I^{I}$ is the inertia tensor of body $I$ in its body-fixed frame, $\Omega^{I}$ is its angular velocity vector, $T_{i j}^{I}$ is the attitude matrix of body $I$ mapping its body-fixed frame to the inertial frame, $\epsilon_{i j k}$ is the skew-symmetric 3 -tensor (with $\epsilon_{123}=1$ ) that defines the cross product, $\mathcal{G}$ is the universal constant of gravitation, $B^{I}$ signifies the mass distribution of the body with differential mass element $d m^{I}, \rho^{I}$ is the location of that mass element in the $I$ th body frame, and $U$ is the mutual gravitational potential between the bodies. The quantities $F_{i}$ and $M_{i}^{I}$ represent the external force and moment, respectively, acting on these systems. These external perturbations generally arise from the gravitational attraction of a planet or the sun, and from radiation induced forces acting on the bodies. Dots over a variable denote time derivatives, subscripts on all variables except $U$ denote vector, matrix or tensor elements, and we assume the Einstein summation convention. A subscript on $U$ denotes partial differentiation. The most difficult item to compute in the above equations of motion is the mutual force potential between the two bodies. Werner \& Scheeres (2005) summarize the literature on this problem and provides a novel and efficient method for evaluation of the mutual potential given standard polyhedral shapes of the bodies.

In the absence of external perturbations, under their self-dynamics, the binary system will exhibit the classical constraints of conservation of energy and conservation of total angular momentum.

For the total energy we have the following scalar quantity

$$
E=\frac{1}{2}\left(I_{i j}^{1} \Omega_{i}^{1} \Omega_{j}^{1}+I_{i j}^{2} \Omega_{i}^{2} \Omega_{j}^{2}\right)+\frac{1}{2} \frac{M_{1} M_{2}}{M_{1}+M_{2}} \dot{r}_{i} \dot{r}_{i}-U\left(r, T^{1}, T^{2}\right)
$$

To properly consider evolutionary behavior of a binary system we must also consider the self-potentials of each body, accounting for the ability of a system to absorb energy into changes in the mass distribution, or shape, of each of the bodies Scheeres (2004), such distortions are also associated with the dissipation of energy. Thus, conservation of energy assumes that the shapes of each body remain fixed, or rigid.

The total angular momentum of the system can be denoted as the following vector

$$
K_{i}=\sum_{I=1}^{2} T_{i j}^{I} I_{j k}^{I} \Omega_{k}+\frac{M_{1} M_{2}}{M_{1}+M_{2}} r_{j} \dot{r}_{k} \epsilon_{i j k}
$$

and is conserved even in the presence of body deformations. In that case the inertia tensors of the bodies may shift as well.

The conservation of these quantities plays an important role in defining and determining the dynamics of a binary asteroid without external perturbation. External 
perturbations can provide changes in the total conserved quantities, allowing the system to evolve over time.

\section{An Ideal Model}

The above relations and definitions can be applied to arbitrary binary systems. However, such detailed models are only in existence for very few binary systems, and most binaries are only known by the grossest properties such as their elongation, apparent diameter, and light-curve derived spin rates (Pravec et al. (2006)). Given this, and given the measured properties of known asteroids, it makes sense to define a simplified model for a binary system. Our basic model will be comprised of two ellipsoidal bodies, with the mutual potential defined by an expansion up to second order only. If one of these bodies is a sphere the problem has been called the Sphere Restricted Full 2-body problem in Scheeres (2002b) or more recently the name "Kinoshita Problem" has been proposed in Breiter et al. (2005) due to the first studies of this problem being made in Kinoshita (1972).

\subsection{Ideal Physical Model of Binaries}

The morphology of most binaries can be "fit" by a relatively few simple observations on their geometry and configuration. We should note that these observations are due, in part, to a dearth of high resolution models of most binaries, and that there are special cases known which may violate each of these assumptions.

- Primary shape is oblate

- Primary rotation rate is rapid and at or near the surface disruption limit

- Secondary shape is elongate

- Secondary rotation is synchronous with orbit

- Mutual orbit is near-circular with inclination near zero

Although there are notable exceptions, the above geometrical features appear to be representative of most NEO binary systems, and are what should be explored generically first. Indeed, the KW4 binary system fits with this ideal model very well.

\subsection{Mutual Potential}

The general mutual potential between two bodies can be stated in a simplified form if we apply MacCullagh's formula to each body. Following Maciejewski (1995) we can develop an explicit formula for the mutual potential between two mass distributions at the second order:

$$
U=\frac{\mathcal{G} M_{1} M_{2}}{\sqrt{r_{k} r_{k}}}\left[1+\frac{1}{2 r_{k} r_{k}}\left(I_{i i}^{1}+I_{i i}^{2}\right)-\frac{3}{2\left(r_{k} r_{k}\right)^{2}}\left(I_{i j}^{1}+I_{i j}^{2}\right) r_{i} r_{j}\right]
$$

We will use this basic formula for the mutual potential in our following discussions.

\subsection{Mechanics of the Ideal Binary Model}

We can separate the internal motion of a binary system, the motion only attributable to the mutual interaction between the two bodies, into two portions: relative orbit and angular evolution. The relative orbit evolution involves the planar orbit of the system and can be described by the classical orbit elements of semi-major axis, eccentricity, argument of periapsis and mean motion. The angular evolution couples orbital and rotational dynamics together and considers the rotational angular momentum of the primary and secondary and the angular momentum vector of the relative orbit, relating to the inclination and longitude of ascending node. 
For each case we can identify a minimum energy configuration for the system, and the dynamics for deviations from these configurations. In the following we define each of these minimum energy configurations and describe the motion in their vicinity.

\subsubsection{Relative Orbit Dynamics}

For the ideal model the minimum energy configuration of the binary system has the secondary in a constant synchronous rotation and the orbit and primary angular momentum vectors aligned. This model fits the basic observational constraints for binary systems. The relative orbit describes a circular path, but the system is not in an osculating circular orbit in general. This is a somewhat subtle point, but can be understood by considering the osculating orbit elements of a simple binary system comprised of a central sphere and a smaller ellipsoidal body. Let us align the ellipsoid in a relative equilibrium with its longest axis pointing towards the sphere and rotating at the necessary rate to balance gravitational attractions. Due to the non-point mass mutual attraction of the two bodies the rotation rate differs from a Keplerian orbit, inducing a semi-major axis not strictly equal to the radius of the orbit. This in turn implies that the orbit has a non-zero eccentricity, with the result that the system has a constant true anomaly equal to 0 or 180 degrees, and a precessing argument of periapsis with period equal to the orbit period.

For the relaxed model the motion is indistinguishable from a classical circular orbit, however when the system is perturbed from this relaxed configuration the resulting dynamics will differ from a slightly eccentric Keplerian orbit. This can be shown in Fig. 1 which shows the eccentricity vector for a binary system comprised of a spherical primary and a non-symmetric secondary body nominally in a synchronous orbit. The relative equilibrium and periapsis libration curves correspond to the above situation, where the true anomaly librates about 0 degrees and the argument of periapsis circulates with a period equal to the orbit period. The circulating periapsis curve is sufficiently excited to break out of this configuration and has its true anomaly circulating and its argument of periapsis moving in a more traditional manner with a secular drift. This is an interesting issue that still requires study, and is relevant as for a general system the primary is not rotationally symmetric, and the orbit and angular evolution of the bodies will have small scale fluctuations about the synchronous state. If the relative equilibrium is energetically stable, these will only lead to small scale deviations from the minimum energy state.

\subsubsection{Angular Momentum Dynamics}

The dynamics of the orbit and primary rotational angular momentum vectors are dominated by conservation of angular momentum. Assuming a mild rotational asymmetry for the primary, the precession of the primary rotation pole and the orbit plane are locked to each other (Kinoshita (1972)). In general the primary and the orbit will lock up the vast majority of the system's angular momentum with the secondary's rotational angular momentum contributing a negligible component. Let the magnitude of the total primary rotational angular momentum equal $H=I_{3}^{1} \Omega_{3}$ and the magnitude of the orbital angular momentum equal $G=\frac{M_{1} M_{2}}{M_{1}+M_{2}} \sqrt{\mathcal{G}\left(M_{1}+M_{2}\right) a}$. Then due to the secondary's assumed on-average synchronous motion and the primary's assumed modest equatorial ellipticity, the respective magnitudes of the primary rotational angular momentum, $H$, and the orbit angular momentum, $G$, are constant on average. Denote the total angular momentum vector to be aligned with the inertial $z$-axis with a magntiude $K$. Let the angle between the primary angular momentum vector and the $z$-axis be $\delta$. Let the angle between the orbit angular momentum and the $z$-axis be $\iota$. Let the sum of the two angles be the mutual obliquity: $\Delta=\delta+\iota$. The inclination and obliquity have only small fluctuations from 


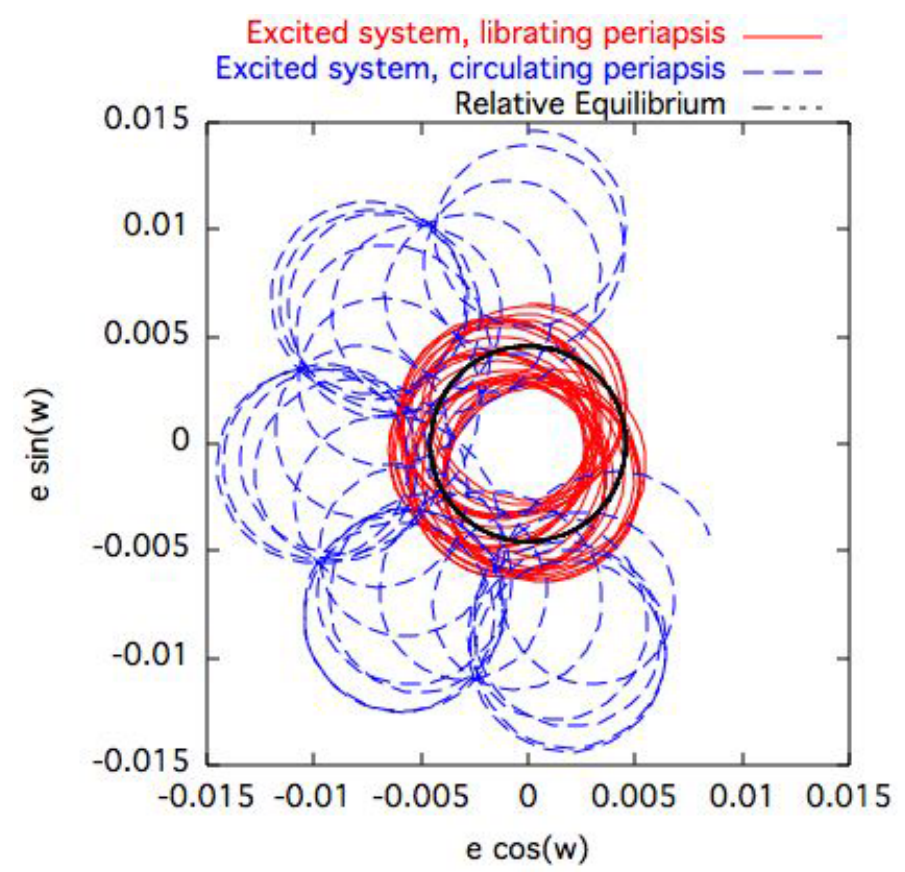

Figure 1. Plot of the eccentricity vector of a binary system at various levels of excitement.

their initial values, so the angular momentum vectors trace out cones in inertial space (Fig. 2).

The following constraints from conservation of total angular momentum then apply, assuming we can ignore the contribution of the secondary rotational angular momentum to the system.

$$
\begin{aligned}
K & =H \cos \delta+G \cos i \\
H \sin \delta & =G \sin i \\
\Delta & \sim \text { Constant }
\end{aligned}
$$

These constraints enforce the following geometry on the system, to within the magnitude of the secondary angular momentum (Scheeres et al. (2006)):

- The primary, orbit and total angular momentum lie in a plane with the total angular momentum lying in between the others.

- The precession period and direction of the orbit plane must equal the precession period of the primary rotation pole.

- The orbit pole traces out a cone with half angle: $\sim(G / K) \Delta$

- The primary pole traces out a cone with half angle: $\sim(H / K) \Delta$

- The minimum energy configuration is $\Delta=0$, as this forces the two spin rates associated with $H$ and $G$ to be minimized, thus minimizing kinetic energy.

By definition, the secondary orientation is locked into a Cassini state, driven by the difference between the orbit and primary angular momentum. If the system has no fluctuations due to non-rotational symmetry, then the attitude of the secondary will be locked in a constant orientation. Due to asymmetries in the system the secondary attitude will in general fluctuate about a mean Cassini state for that system Colombo (1966). 
These secular dynamics can also be predicted from basic analysis of the mean orbit plane precession formula and the mean primary spin-axis precession formula. When carried out in detail each of these analyses predict the same mean precession rate, equal to:

$$
\dot{\psi}=-\frac{3 \sqrt{\mathcal{G}\left(M_{1}+M_{2}\right)}}{\sqrt{a^{3}}} \frac{I_{a}-I_{t}}{a^{2}} \cos \Delta
$$

where $I_{a}$ and $I_{t}$ are the axial and transverse moments of inertia from the assumed rotationally symmetric primary.

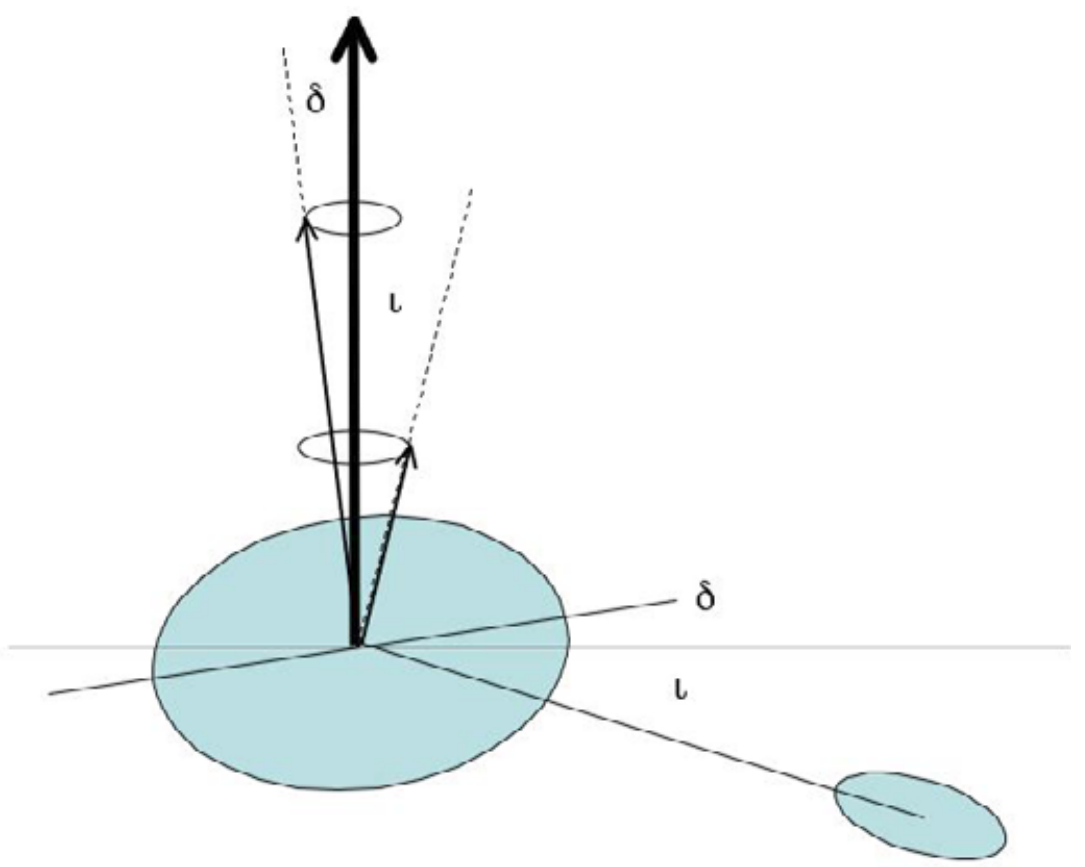

Figure 2. The diagram shows the path followed by the evolving angular momentum vectors. The large vertical arrow represents the total angular momentum, the smallest arrow represents the orbit angular momentum, which has an angle $i$ with the total angular momentum, and the other arrow represents the primary's angular momentum, which has an angle $\delta$ with the total angular momentum. The secondary's rotational angular momentum is too small to show in general.

\subsection{Internal Stability}

An interesting and important topic for the ideal binary model is the stability of the proposed relative equilibrium configuration. When the formal stability of these configurations are determined, only those binary systems which match the generally observed binary morphology are stable, thus potentially explaining why other binary morphologies are not found. In the following we define internal stability as the stability of the system without external perturbation. Clearly, if a system is not internally stable it is not likely to be found in nature, independent of the external perturbations acting on it.

There are at least three different relevant definitions of stability for a binary asteroid:

- Stability against escape: Can or will the two components escape each other?

- Stability against impact: Can or will the two components impact each other?

- Orbit/Configuration stability: Can the current orbit or configuration of the system persist? 
Each of these stability types can be evaluated using well-defined mathematical techniques and evaluations Scheeres (2002b), Scheeres (2006). These conditions can be reduced to a few basic observations.

First, configuration or orbit stability reduces to the energetic stability of the relative equilibrium. An equilibrium configuration is energetically stable if it inhabits the minimum energy configuration possible for that system at a given system angular momentum. We note that there are a number of possible relative equilibrium for a binary system that may be spectrally stable, but there is only one that ever has, in addition, energetic stability. These invariably have the minimum moment of inertia principal axis directed towards the primary, although for non-symmetric bodies the axis does not point precisely at the body Scheeres (2006). This generalizes to the common gravity gradient orientation of Earth and natural satellites. Its important to note that for mass distributions between the two binary bodies that are not dominated by the primary, that these configurations can be unstable. The classical example being that a particle placed in a relative equilibrium along the minimum moment of inertia axis of a massive ellipsoid is always unstable. Thus, there is a transition in these configurations from unstable to stable as the primary body mass becomes dominant. In Figs. 3 and 4 these stability curves are presented for two different ellipsoid-sphere relative equilibrium configurations. Each point on these diagrams defines a relative equilibrium between the sphere and ellipsoid, with the minimum moment of inertia axis of the ellipsoid pointing at the sphere and the system rotating about the maximum moment of inertia. Points above the stability limit line are energetically stable, and points below are not. The equal density distance is defined as the distance between the mass center of the ellipsoid and a sphere assuming an equal density for each body. Thus, this distance is only a function of the mass ratio between the bodies. Note that the distance has been normalized by the long axis of the ellipsoid. We note that even though the two ellipsoids have significantly different shapes, the qualitative features of their stability limit lines are similar. This indicates that sphericity of the primary is an essential ingredient for the overall stability of the given configuration.

Stability against mutual escape is ensured by the total energy being negative, $E<0$, while if $E>0$ it is possible for the system to escape. Note that most binaries technically violate this due to the extremely rapid rotation rate of the primary. However, if we note that the mass distribution of a spinning spheroidal body cannot, in general, interact with the orbital system we should remove this energy from consideration, which in general will reduce the energy of the system considerably. This reduced or "free" energy is what is shown in Figs. 3 and 4. We note that all binary systems whose relative equilibrium has a positive free energy are also unstable, this result holds across all parameter values for this ideal system. Having a positive free energy is only a necessary condition for the system to have a mutual escape, and it is possible for a positive energy system to also suffer impact.

Stability against impact is ensured by the relative equilibrium of the system being stable, or by the total angular momentum being high enough. Note that most binaries satisfy this formally, again due to the rapid rotation of the primary. In Scheeres (2002b) a specific condition for impact stability of a system is derived. A simpler observation can be made with the aid of the stability diagrams above. Here we note that whenever a relative equilibrium configuration is unstable, then there will exist unstable manifolds that travel from that relative equilibrium configuration, one of which increases the distance between the bodies and the other decreases the distance, this latter generally intersecting with the surface of the body. Thus, configuration instability implies that a pathway for the bodies to impact also exists. While the ideal situation has the system initially placed in 


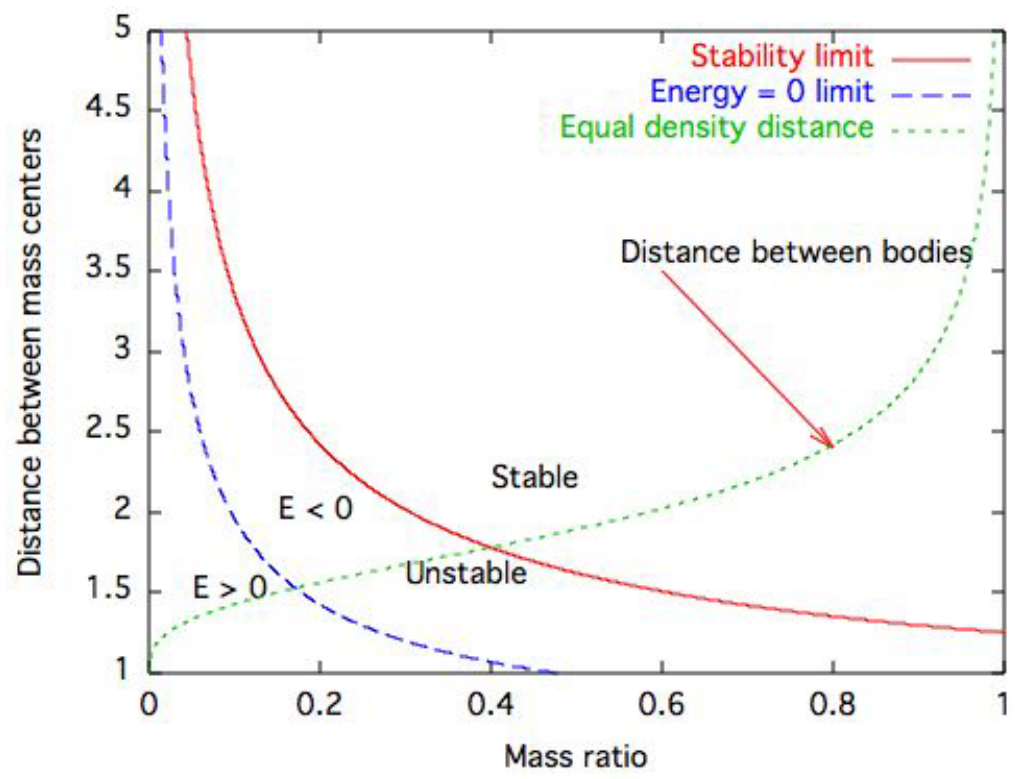

Figure 3. Stability diagram for a sphere-ellipsoid system in relative equilibrium, ellipsoid semi-major axes of 1:0.9:0.8. A mass fraction of 0 represents a particle about a massive ellipsoid, while a mass fraction of 1 represents an ellipsoid with negligible mass about a massive sphere.

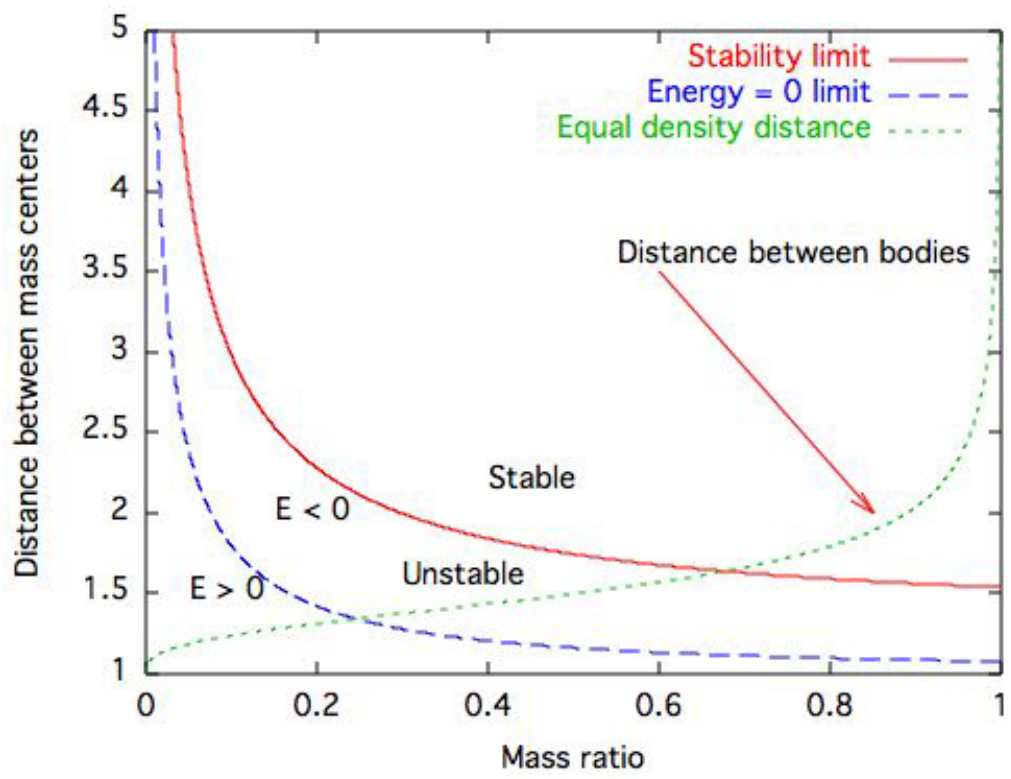

Figure 4. Stability diagram for a sphere-ellipsoid system in relative equilibrium, ellipsoid semi-major axes of 1:0.5:0.25.

a relative equilibrium, for a realistic system the "initial conditions" will be more general, but the existence of these pathways to impact will still hold and make it more likely that the system can transition into an impact, perhaps forming a contact binary. As the relative equilibrium approaches the stability limit, these unstable manifolds may no 
longer lead to mutual impacts, the limit for when this occurs has yet to be studied in detail, however.

From these stability results we can infer a number of general results on what sort of binary systems can survive naturally. We note that systems with elongate primaries are generally unstable at smaller separation distances and hence should either lead to mutual escape or impact. Systems with spheroidal primaries are more prone to have stable configurations for their orbiting member and should persist, which is in agreement for the ideal model of binaries inferred from observations. We also note that almost any binary system with sufficient separation can be stable, including elongate primaries. The apparent lack of such binary systems implies that, at some point of their formation or evolution, binary asteroids may reside in a close configuration which would then destroy such systems.

\section{External Perturbations}

External perturbations to an NEO binary system can arise from several sources, each of which will have a different signature. The perturbations we will consider, and the ones most relevant for these systems, are due to planetary flybys, solar gravity effects, and solar radiation effects.

\subsection{Gravitational Perturbations}

While the effect of planetary flybys and perihelion passages are similar in that in both cases the perturbations arise from a combination of gravity and centripetal accelerations, we find that the net effect of these flybys are quite different. Still, we can begin our analysis with a common investigation and later separate the effect of these interactions. Following from Marchal (1990) and Scheeres \& Marzari (2002) we can define a modified form of the elliptic/hyperbolic Hill 3-body problem that only incorporates the effect of gravity. The main effect of the gravitational perturbation during a periapsis passage is characterized by the radial location of the libration point as measured from the binary center of mass towards or away from the sun, equal to $x_{P}=\left(\frac{\mu}{3 \mu_{P}}\right)^{1 / 3} q$, where $\mu$ and $\mu_{P}$ are the gravitational parameters of the binary system and the perturbing body, respectively, and $q$ is the periapsis radius, or distance between the binary center of mass and the perturbing body at closest approach. The ratio between the binary orbit semimajor axis and the libration point distance $x_{P}$ controls the strength of the perturbation, and whether or not the perturbation can disrupt the binary during closest approach. A basic result is that if the relationship $a / x_{P}<1 / 3$ holds, the binary cannot be directly disrupted due to the flyby Marchal (1990), although it can be significantly perturbed and may impact following closest approach.

Another important consideration is the angular rate of the flyby, specifically the comparison between the angular rate of the binary relative to the perturbing body and the angular rate of the binary orbit itself. During perihelion passage the binary system will be traveling at a rate $\dot{\nu}=\sqrt{\mu_{P}(1+e) / q^{3}}$, where $e$ is the eccentricity of the flyby orbit and will be greater than one for a planetary flyby (i.e., be a hyperbolic orbit) and less than one for a solar close approach. The angular orbital rate of the binary is approximately equal to $n=\sqrt{\mu / a^{3}}$. The ratio between these two is important as it controls whether the perturbation is "impulsive", i.e. if it acts fast relative to the orbit rate of the binary system, or whether it must be averaged over several binary orbit periods. As we will demonstrate with some simple examples later these lead to qualitatively different effects on the orbit. Taking this ratio we see that the result is a function of the libration 
point at periapsis, $x_{P}$, defined earlier:

$$
\frac{\dot{\nu}}{n}=\sqrt{1+e}\left(\frac{a}{x_{P}}\right)^{3 / 2}
$$

When this ratio is small, the effect of the perturbing gravity will be averaged over more rotation angle of the binary, while when it is large the binary orientation will be approximately fixed during the fly-by and the perturbing gravity will act impulsively.

\subsubsection{Planetary flybys}

For planetary flybys the eccentricity equals $e=1+q V_{\infty}^{2} / \mu_{P}$ and is greater than 1 . This, combined with the possibility of very close flyby distances which makes the ratio $a / x_{P}$ relatively larger, will lead to an impulsive nature for these interactions. Such a flyby can inject energy and angular momentum into the binary system nearly instantaneously, leading to escape, impact, or major perturbation Farinella (1992). The expected signature of a close approach that doesn't destroy a binary will be a highly randomized system until relaxation effects come into play. We note that this process may also be more efficient at disrupting binaries than at creating binaries, as the disruption distance is greater than the creation distanceRichardson \& Walsh (2007). In Fig. 5 we show some example perturbations to a binary system subject to a planetary flyby. The binary model used in these simulations is the Keplerian binary so that the effect of the external perturbation can be clearly seen without any other perturbations. It is important to note that even if the flyby does not directly destroy the binary, it is possible for the perturbed binary to run afoul of the solar perturbation due to an increase in semi-major axis, and become subject to such solar perturbations.

\subsubsection{Solar gravity effects}

Solar gravity effects are only active for asteroids with perihelia low enough such that the ratio $a / x_{P}$ grows larger. For these cases the solar perturbation can be a significant source of excitation, however. As the eccentricity of the orbit is less than one by definition, and as the ratio $a / x_{P}$ tends to be small, $\dot{\nu} / n \ll 1$ and the gravitational interaction generally occurs over a period of many binary revolutions, and hence the net effect is much more subdued than a planetary flyby. Since the effect is active for an extended period of time, however, it can create significant excitation of a system without disrupting it. Applying the above relations we find that for a binary system with $\mu=10^{-7} \mathrm{~km}^{3} / \mathrm{s}^{2}$ the semi-major axis relation becomes $a<32 q$ where $q$ is measured in astronomical units (AU). Thus, for a typical separation of less than 5 kilometers, perihelion must come down to $0.15 \mathrm{AU}$ before the system can be completely disrupted by the sun. Prior to this, however, the system is subject to perturbations during each perihelion passage. The nature of these perturbations are much different than planetary flybys as the significant perturbation lasts over many binary orbit revolutions. Thus there is a large degree of averaging that occurs during perihelion passage which changes the effect of the perturbation, seen in Fig. 6 . We note that the perihelion passage is able to measurably shift the system inclination. Also, the excitation of the eccentricity can stimulate internal motions that leave the binary in an excited state, even if the eccentricity reduces back to a small value as is the case for the simulations shown here (due to the nominal binary orbit being modeled as a Keplerian 2-body problem).

\subsection{Solar radiation effects}

Incident sunlight carries momentum that is transfered to the system and reemitted by reflection and thermal re-emission. This momentum flux can cause changes in the rotation 

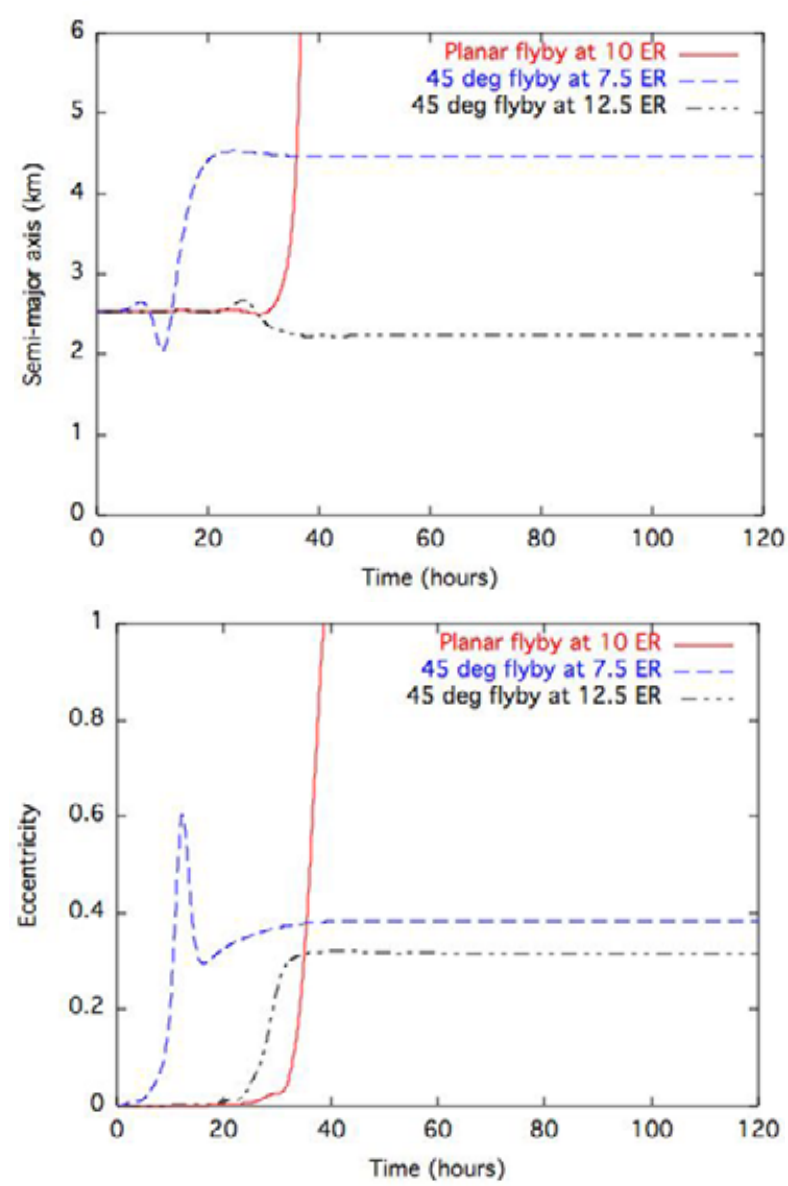

Figure 5. Evolution of the semi-major axis, eccentricity and inclination of a binary system during an Earth flyby

state and mutual orbit of the system. The radiation acts independently on each body, but can be coupled by the dynamical reaction of each body. There are two main effects, a force and a torque acting on each component of the system. The study and understanding of the effects of these forces and torques on binary systems is one of the most pressing issues for this field of study.

\subsubsection{Solar radiation pressure force}

The force acting on the primary will mainly affect the heliocentric trajectory of the system. In particular, the thermal characteristics of the primary become important as it is only the reemission of radiation transverse to the orbit that will have a measurable effect on the heliocentric trajectory, called the Yarkovsky effect, a topic that has been studied extensively in Bottke et al. (2002) and analyzed specifically for binary asteroids in Vokrouhlický et al. (2005). The force acting on the secondary will affect the mutual orbit of the binary system as this will act as a small non-gravitational force acting on the system. Any asymmetry in the net force acting on the body can cause a small but finite net torque to be delivered to the orbit and cause the orbit to grow or shrink in time, what is called the Binary YORP (BYORP) effect Ćuk \& Burns (2005). To date the only detailed analysis of the long-term implications of this effect is in Cuk \& Burns 

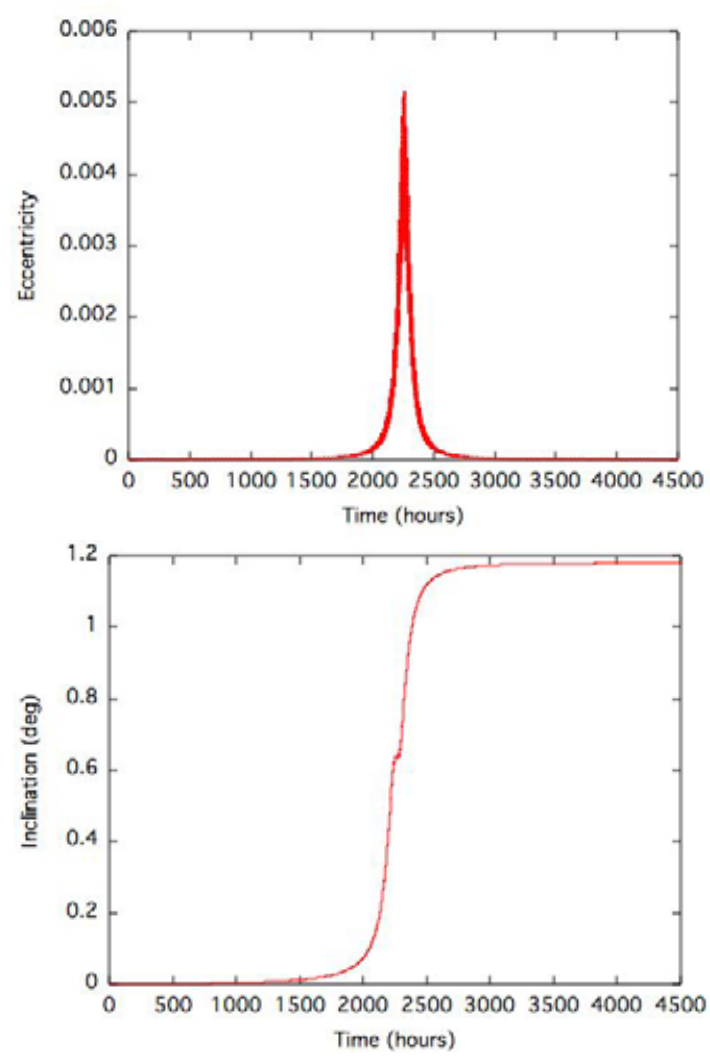

Figure 6. Evolution of the semi-major axis, eccentricity and inclination of a binary system during a perihelion passage

(2005) where they provide a first-order averaging analysis of this effect on binary orbits and predict that extremely rapid migration of these orbits may occur, with disruption or impact on the order of thousands of years. The analysis in that paper does make some simplifying assumptions, mainly that the net differential force acting on the secondary can be modeled as an averaged, constant force over long periods of time. This may be a reasonable assumption but the extremely short lifetimes are difficult to reconcile with the number of binary objects that have been observed in the NEO population, and imply an extremely fast production rate of binaries. The analysis by Vokrouhlický et al. (2005) investigates the effect of the Yarkovsky force on binary asteroids over shorter time spans, and also provide an analysis of the Yarkovsky-Schach effect, which arises due to the primary eclipsing the secondary. A deeper understanding of these dynamics over long time spans is a pressing topic in this area.

\subsubsection{Solar radiation pressure torques}

The effect of solar insolation on the rotation states of the primary and secondary can also be significant for the evolution of the system. Asymmetries in either of the bodies can lead to a net torque acting on them, with the rotational acceleration and obliquity of the bodies modified Rubincam (2000), this effect has been termed the YORP effect in the literature.

YORP can also cause the primary to either increase its spin rate or decrease it over time, pumping angular momentum into or out of the system. If it increases, it is possible for material to be spun off the surface of the primary, which would then transfer angular 
momentum to the orbit and cause the system to expand. If it decreases, it could eventually lead to a mutually synchronous system. YORP acceleration acting on the secondary will bias its attitude relative to the secondary-primary line, allowing for the transfer of angular momentum to the orbit. For a positive torque, this would lead to a growth in the orbit and, ultimately, in a decrease in the spin rate of the secondary. A negative YORP torque on the secondary should lead to a shrinking in the orbit and a speed up in the spin rate of the secondary. A possible characteristic of systems subject to YORP may be the rapid spin of the primary at or near the disruption limit. None of the other external perturbations can, as easily, lead to this state.

\section{Conclusions}

This paper reviews current literature on the dynamics of binary asteroids and presents a summary of what is currently known and hypothesized on these systems. The general governing equations of motion and constraints are stated. A simplified, but non-trivial, model for the study of binary asteroids is proposed and discussed. A discussion on the stability of binary asteroids is given, and we note that binary systems that should be unstable at close separation distances are not found in nature, implying that binary asteroids may go through a close configuration at some point in their lifetime. Finally, we discuss the main features and effects of the main external perturbations that act on binary asteroids in the near-Earth population.

\section{Acknowledgements}

This research was supported by a grant from NASA's Planetary Geology and Geophysics Program.

\section{References}

Bottke, W.F., Vokrouhlický, D., Rubincam, D.P. \& Brož, M. 2002, in W.F. Bottke, A. Cellino. P. Paolicchi \& R. Binzel (eds.), Asteroids III (Arizona University Press, Tucson), p. 395

Breiter, S., Melendo, B., Bartczak, P. \& Wytrzyszczak, I. 2005, Astron. Astrophys. 437, 753

Chauvineau, B., Farinella, P. \& Mignard, F. 1993, Icarus 105, 370

Colombo, G. 1966, Astron. J. 71, 891

Ćuk, M. \& Burns, J.A. 2005, Icarus 176, 418

Farinella, P. 1992, Icarus 96, 284

Hamilton, D.P. \& Burns, J.A. 1991, Icarus 92, 118

Kinoshita, H. 1972, Publ. Astron. Soc. Japan 24, 423

Maciejewski, A.J. 1995, Celest. Mech. Dyn. Astron. 63, 1

Marchal, C. 1990, The Three-Body Problem, Elsevier

Ostro, S.J., Margot, J.-L., Benner, L.A.M., Giorgini, J.D., Scheeres, D.J., Fahnestock, E.G., Broschart, S.B., Bellerose, J., Nolan, M.C., Magri, C., Pravec, P., Scheirich, P., Rose, R., Jurgens, R.F., Suzuki, S. \& DeJong, E.M. 2006, Radar Imaging of Binary Near-Earth Asteroid (66391) 1999 KW4, Science, in press

Pravec, P., Scheirich, P., Kusnirák, P. \& Sarounová, L. 2006, Icarus 181, 63

Richardson, D. \& Walsh, K. 2007, this volume

Rubincam, D.P. 2000, Icarus 148, 2

Scheeres, D.J. 1994, Icarus 110, 225

Scheeres, D.J., Ostro, S.J., Hudson, R.S. \& Werner, R.A. 1996, Icarus 121, 67

Scheeres, D.J., Ostro, S.J., Hudson, R.S., DeJong, E.M. \& Suzuki, S. 1998, Icarus 132, 53

Scheeres, D.J. \& Marzari, F. 2002, J. Astronautical Sciences 50, 35

Scheeres, D.J. 2002, Icarus 159, 271

Scheeres, D.J. 2002, Celest. Mech. Dynam. Astr. 83, 155 
Scheeres, D.J. 2004, Celest. Mech. Dynam. Astr. 89, 127

Scheeres, D.J. \& Bellerose, J. 2005, Dynamical Systems: An International Journal 20, 23

Scheeres, D.J. 2006, Celest. Mech. Dynam. Astr. 94, 317

Scheeres, D.J., Fahnestock, E.G., Ostro, S.J., Margot, J.-L., Benner, L.A.M., Broschart, S.B., Bellerose, J., Giorgini, J.D., Nolan, M.C., Magri, C., Pravec, P., Scheirich, P., Rose, R., Jurgens, R.F., Suzuki, S. \& DeJong, E.M. 2006, Dynamical Configuration of Binary NearEarth Asteroid (66391) 1999 KW4, Science, in press

Vokrouhlický, D., Čapek, D., Chesley, S.R. \& Ostro, S.J. 2005, Icarus 179, 128

Werner, R.A. \& Scheeres, D.J. 2005, Celest. Mech. Dynam. Astr. 91, 337 